\title{
Diferentes arranjos de semeadura e genótipos de amendoim nas condições de Campo Verde-MT
}

\author{
Submetido - 06 jun. 2021 \\ Aprovado - 30 jul. 2021 \\ Publicado - 30 set. 2021
}

http://dx.doi.org/10.52755/sas.v.2i(edesp1)114

\begin{abstract}
Discente de Agronomia do IFMT Campus São Vicente - Centro de Referência de Campo Verde, Campo Verde, MT. E-mail: daniele.fernandes@yahoo.com.
\end{abstract}

Alexandre Caetano Perozini iD Docente do IFMT Campus São Vicente - Centro de Referência de Campo Verde, Campo Verde, MT. E-mail: alexandre.perozini@svc.ifmt.edu.br.

Programa de Melhoramento do Amendoim - Embrapa, Santo Antônio de Goiás, GO. E-mail: jair.heuert@embrapa.br.

Maxsuel Antonio Rodrigues (D)

Discente de Agronomia do IFMT Campus São Vicente - Centro de Referência de Campo Verde, Campo Verde, MT. E-mail: maxsuel12ar@gmail.com.

Maxuel Fellipe Nunes Xavier (iD)

Discente de Agronomia do IFMT Campus São Vicente - Centro de Referência de Campo Verde, Campo Verde, MT. E-mail: maxuelfellipe90@gmail.com.

Taís de Moraes Falleiro Suassuna (iD

Programa de Melhoramento do Amendoim - Embrapa, Santo Antônio de Goiás, GO. E-mail: tais.suassuna@embrapa.br.

\section{RESUMO}

O presente trabalho teve como objetivo avaliar os diferentes arranjos de semeadura e genótipos de amendoim nas condições de Campo Verde-MT. O experimento foi desenvolvido na área experimental do Instituto Federal de Educação, Ciência e Tecnologia de Mato Grosso Campus São Vicente, Centro de Referência de Campo Verde, localizado no município de Campo Verde - MT, no ano agrícola 2020/21. A semeadura foi realizada de forma manual no dia 16 de novembro de 2020. O delineamento experimental utilizado foi em blocos casualizados, com quatro repetições, em esquema fatorial 4 x 3, sendo 4 genótipos (BRS 421 OL, BRS 423 $O L$, BRS 425 OL e $1952 \mathrm{OL}$ ) e 3 arranjos de semeadura $\left(0,73 \mathrm{~m}\right.$ x 0,17 m e 15 plantas. $\mathrm{m}^{-1} ; 0,70 \mathrm{~m}$ e 20 plantas. $m^{-1} ; 0,90$ m e 20 plantas. $\left.m^{-1}\right)$. A colheita foi realizada aos 133 dias após o plantio (DAP), onde foram avaliados severidade de mancha preta (notas), massa de 100 grãos (g) e produtividade de vagens (kg.ha-1 e sacas.alqueire ${ }^{-1}$ ). Nas condições experimentais em que foi desenvolvida esta pesquisa, pode-se concluir que a os arranjos de semeadura não influenciam de forma significativa nas variáveis. No entanto, os genótipos influenciaram na severidade e produtividade, sobressaindo-se com o BRS 421 OL, BRS 423 OL e BRS 425 OL. Sobretudo, mais estudos são recomendados para confirmação dos resultados obtidos.

Palavras-chave: Arachis hypogaea L.; Espaçamentos; Amendoim em Mato Grosso; Programa de Melhoramento.

Different sowing arrangements and peanut genotypes under the conditions of Campo Verde-MT

\section{ABSTRACT}

Este é um trabalho de acesso aberto e distribuído sob os Termos da Creative Commons Attribution Attribution-NonCommercial-ShareAlike 4.0 International. 
The present work aimed to evaluate the different sowing arrangements and peanut genotypes in Campo Verde-MT conditions. The experiment was carried out in the experimental area of the Federal Institute of Education, Science and Technology of Mato Grosso - Campus São Vicente, Reference Center of Campo Verde, located in the municipality of Campo Verde - MT, in the agricultural year 2020/21. Sowing was performed manually on November 16, 2020. The experimental design used was a randomized block design, with four replications, in a $4 \times 3$ factorial scheme, with 4 genotypes (BRS 421 OL, BRS 423 OL, BRS 425 OL and 1952 OL) and 3 sowing arrangements $\left(0.73 \mathrm{~m} \times 0.17 \mathrm{~m}\right.$ and 15 plants. $\mathrm{m}^{-1} ; 0.70 \mathrm{~m}$ and 20 plants. $\mathrm{m}^{-1} ; 0.90 \mathrm{~m} \times 20$ plants. $\left.{ }^{-1}\right)$. Harvest was carried out 133 days after planting (DAP), where black spot severity (grades), 100 grain mass (g) and pod yield (kg.ha-1 and sacks.alqueire ${ }^{-1}$ ) were evaluated. In the experimental conditions in which this research was developed, it can be concluded that the sowing arrangements do not significantly influence the variables. However, the genotypes influenced the severity and productivity, standing out with the BRS $421 O L, B R S 423 O L$ and BRS $425 \mathrm{OL}$. Above all, further studies are recommended to confirm the results obtained.

Keywords: Arachis hypogaea L.; Spacing; Peanuts in Mato Grosso; Breeding Program.

\title{
Diferentes arreglos de siembra y genotipos de maní en las condiciones de Campo Verde-MT
}

\author{
RESUMEN
}

El presente trabajo tuvo como objetivo evaluar los diferentes arreglos de siembra y genotipos de maní en condiciones de Campo Verde-MT. El experimento se realizó en el área experimental del Instituto Federal de Educación, Ciencia y Tecnología de Mato Grosso - Campus São Vicente, Centro de Referencia de Campo Verde, ubicado en el municipio de Campo Verde - MT, en el año agrícola 2020/21. La siembra se realizó manualmente el 16 de noviembre de 2020. El diseño experimental utilizado fue un diseño de bloques al azar, con cuatro repeticiones, en un esquema factorial 4 x 3, con 4 genotipos (BRS 421 OL, BRS 423 OL, BRS 425 OL y 1952 OL) y 3 arreglos de siembra $\left(0,73 m\right.$ x 0,17 m y 15 plantas. $m^{-1} ; 0,70$ m y 20 plantas. $m^{-1} ; 0,90 m$ m 20 plantas. $\left.m^{-1}\right)$. La cosecha se realizó 133 días después de la siembra (DAP), donde se evaluó la severidad de la mancha negra (grados), masa de 100 granos (g) y rendimiento de vaina (kg.ha-1 $y$ sacks.alqueire $\left.{ }^{-1}\right)$. En las condiciones experimentales en las que se desarrolló esta investigación, se puede concluir que los arreglos de siembra no influyen significativamente en las variables. Sin embargo, los genotipos influyeron en la severidad y productividad, destacándose con el BRS 421 OL, BRS 423 OL y BRS 425 OL. Sobre todo, se recomiendan más estudios para confirmar los resultados obtenidos.

Palabras clave: Arachis hypogaea L.; Espaciamiento; Maní en Mato Grosso; Programa de Mejoramiento.

\section{Introdução}

O espaçamento de uma cultura é definido pela densidade e disposição de plantas na área cultivada. Entende-se como densidade, o número de plantas por unidade de área, resultante da combinação entre o espaçamento entrelinhas e o número de plantas por metro linear (BERNADES; VICCARIO, 1986). Dentre os fatores que interferem na produtividade de uma cultura, destaca-se a população por afetar diretamente os componentes de produção. Nesse sentido, a fixação desta população 
pode variar em função da cultivar, da época de semeadura, da adubação e da região de cultivo (NAKAGAWA et al., 1983).

O uso de espaçamento adequado de plantas, além de contribuir para maximizar a produtividade, tem efeitos sobre o controle de plantas daninhas, podendo representar uma estratégia importante para utilização de alguns fatores de produção como luz, água e nutrientes. É importante, no entanto, atentar para alguns aspectos que podem auxiliar nas decisões a serem tomadas, como maior ou menor consumo de sementes e realização de tratos culturais (NAKAGAWA et al., 1994).

Nos últimos anos, arranjos de semeadura na cultura do amendoim vêm sendo estudados nas condições edafoclimáticas de Campo Verde-MT, por meio da parceria do Programa de Melhoramento do Amendoim (PMA) da Embrapa com o Instituto Federal de Educação, Ciência e Tecnologia de Mato Grosso - Campus São Vicente, Centro de Referência de Campo Verde (GIRON et al., 2019; GIRON et al., 2020). Com base nestes estudos, observa-se produtividades superiores a estimativa média nacional do cultivo em primeira safra $\left(3.669,0 \mathrm{~kg} \mathrm{ha}^{-1}\right)$, de acordo com os dados da CONAB (2021).

O espaçamento entre linhas simples de $0,70 \mathrm{~m}$ é utilizado na Argentina no cultivo de amendoim (GAMBA; PEDELINI, 2009). Nesse sentido, o espaçamento entre linhas simples de $0,90 \mathrm{~m}$ e linhas duplas de $0,73 \mathrm{~m} \times 0,17 \mathrm{~m}$ são utilizados nos Estados Unidos e no Brasil (HEUERT et al., 2017). Com base nisso, o presente trabalho teve como objetivo avaliar os diferentes arranjos de semeadura e genótipos de amendoim nas condições de Campo Verde-MT.

\section{Material e Métodos}

O experimento foi desenvolvido no ano agrícola 2020/21, na área experimental do Instituto Federal de Educação, Ciência e Tecnologia de Mato Grosso - Campus São Vicente, Centro de Referência de Campo Verde, localizado no município de Campo Verde - MT, cujas coordenadas 
geográficas são $55^{\circ} 10^{\prime} 08^{\prime \prime} \mathrm{W}$ e $15^{\circ} 32^{\prime} 48^{\prime \prime} \mathrm{S}$, com altitude de 736 metros. A semeadura foi realizada de forma manual no dia 16 de novembro de 2020.

O delineamento experimental foi em blocos casualizados, com quatro repetições, em esquema fatorial $4 \times 3$, sendo 4 genótipos (BRS 421 OL, BRS 423 OL, BRS 425 OL e 1952 OL) e 3 arranjos de semeadura $(0,73$ $\mathrm{m} \times 0,17 \mathrm{~m}$ e 15 plantas. $\mathrm{m}^{-1} ; 0,70 \mathrm{~m}$ e 20 plantas. $\mathrm{m}^{-1} ; 0,90 \mathrm{~m}$ e 20 plantas. $\mathrm{m}^{-}$ ${ }^{1}$ ). Todos estes genótipos de amendoim rasteiro foram desenvolvidos pelo PMA. As parcelas eram compostas por duas linhas de três metros de comprimento, com intervalo entre parcelas de dois metros e áreas úteis de $5,4 m^{2}(0,90 \mathrm{~m}$ e $0,73 \mathrm{~m} \times 0,17 \mathrm{~m})$ e $4,2 \mathrm{~m}^{2}(0,70 \mathrm{~m})$.

O manejo fitossanitário da área experimental seguiu as recomendações para a cultura, sendo o controle de plantas daninhas realizado com duas aplicações de herbicida: imazapique $(0,14 \mathrm{~kg}$ p.c.ha-1) nos dias 21/12/2020 e 28/01/2021. O manejo de pragas e doenças foi realizado com oito aplicações de inseticida clorfenapir $\left(0,5 \mathrm{~L}\right.$ p.c.ha $\left.{ }^{-1}\right)$ e fungicidas clorotalonil (1,5 L p.c.ha-1), pyraclostrobina + epoxiconazol $(0,6 \mathrm{~L}$ p.c.ha- ${ }^{-1}$ ) nos dias 10/12/2020, 21/12/2020, 28/12/2020, 08/01/2020, 28/01/2021, 11/02/2021, 28/02/2021 e 11/02/2021.

Com base na necessidade do solo, foi realizado adubação de semeadura de $70 \mathrm{~kg} \cdot \mathrm{ha}^{-1}$ de cloreto de potássio $(\mathrm{KCl})$ e $500 \mathrm{~kg} \cdot \mathrm{ha}^{-1}$ superfosfato simples (SFS) no sulco de plantio. No tratamento de sementes foi utilizado carbendazim, na dose de 0,1 L por $100 \mathrm{~kg}$ de sementes. Foram feitas duas aplicações de $\mathrm{KCl}$, como adubação de cobertura, nos dias 22/12/2020 e 03/01/2021, ambas na dose de $50 \mathrm{~kg}$ ha $^{-1}$ e uma aplicação de gesso agrícola no dia 29/12/2020 na dose de $500 \mathrm{~kg} \mathrm{ha}^{-1}$.

A colheita foi realizada aos 133 dias após o plantio (DAP), onde foram avaliados severidade de mancha preta (notas), massa de 100 grãos (g) e produtividade de vagens ( $\mathrm{kg}^{-h^{-1}}{ }^{-1}$ e sacas.alqueire ${ }^{-1}$ ) mediante a pesagem de vagens e grãos, da área de 3,6 $\mathrm{m}^{2}(0,90 \mathrm{~m}$ e 0,73 $\mathrm{m} \times 0,17 \mathrm{~m}) \mathrm{e}$ $2,8 \mathrm{~m}^{2}(0,70 \mathrm{~m})$ centrais das duas linhas da parcela. 
A severidade de mancha preta (Cercosporidium personatum) foi avaliada usando a escala diagramática da incidência com notas de 1 a 9 ao final do ciclo dos genótipos (SUBRAHMANYAM et al., 1982). Os dados obtidos foram submetidos à análise de variância (Teste $F$ ) e as médias dos tratamentos foram comparadas pelo teste de Scott-Knott a $5 \%$ de probabilidade, por meio do programa computacional ASSISTAT 7.7 (SILVA; AZEVEDO, 2016).

\section{Resultados e Discussão}

Analisando os dados obtidos, observa-se na análise de variância que não houve interação significativa entre os fatores, sendo constatado efeito significativo dos genótipos nas variáveis severidade de mancha preta (notas) e massa de 100 grãos (g) (Tabela 1).

Tabela 1. Resumo da análise de variância para severidade de mancha preta (notas) (SEV), massa de 100 grãos (g) (M100) e produtividade de vagens $\left(\mathrm{kg} \mathrm{ha}^{-1}\right)$ (PROD) em função de diferentes arranjos de semeadura e genótipos de amendoim nas condições de Campo Verde-MT, safra 2020/21.

\begin{tabular}{lccc}
\hline \multirow{2}{*}{ Fontes de variação } & \multicolumn{3}{c}{ Quadrados Médios } \\
\cline { 2 - 4 } & SEV (notas) & M100 $(\mathrm{g})$ & PROD $\left(\mathrm{kg} \cdot \mathrm{ha}^{-1}\right)$ \\
\hline Genótipos (A) & $0,78^{*}$ & $379,17^{\text {ns }}$ & $1.448 .081,95^{*}$ \\
Arranjos de semeadura (B) & $0,23^{\text {ns }}$ & $1,76^{\text {ns }}$ & $4.069 .614,78^{\text {ns }}$ \\
Interação (A x B) & $0,26^{\text {ns }}$ & $6,88^{\text {ns }}$ & $222.272,02^{\text {ns }}$ \\
\hline Coeficiente de variação (\%) & 6,04 & 4,23 & 16,22 \\
\hline * - significativo a 5\% de probabilidade pelo teste de Scott-Knott; ns - não \\
significativo.
\end{tabular}

Constatou maior severidade de mancha preta na linhagem 1952 OL $(8,2)$, diferindo significativamente das notas obtidas nas cultivares BRS 421 OL $(7,6)$, BRS 423 OL $(7,8)$ e BRS 425 OL $(7,8)$ (Tabela 2). De modo geral, ambos os genótipos obtiveram notas superiores a 7,0, demonstrando assim lesões em todas as folhas, porém menos severas no topo (SUBRAHMANYAM et al., 1982). Corroborando com o presente estudo, Xavier et al. (2020), obtiveram as menores notas de severidade na BRS 421 OL, BRS 423 OL e BRS 425 OL, em comparação a 1952 OL, nas condições de Campo Verde-MT. 
Tabela 2. Desdobramento da severidade de mancha preta (notas) e massa de 100 grãos (g) em função dos diferentes genótipos de amendoim nas condições de Campo Verde-MT, safra 2020/21.

\begin{tabular}{ccc}
\hline \multirow{2}{*}{ Genótipos } & Severidade de mancha preta & Produtividade de vagens \\
\cline { 2 - 3 } & (notas) & $\left(\mathrm{kg} \cdot \mathrm{ha}^{-1}\right)$ \\
\hline BRS 421 OL & $7,6 \mathrm{~b}$ & $3.941,7 \mathrm{a}$ \\
BRS 423 OL & $7,8 \mathrm{~b}$ & $3.690,2 \mathrm{a}$ \\
BRS 425 OL & $7,8 \mathrm{~b}$ & $3.886,4 \mathrm{a}$ \\
1952 OL & $8,2 \mathrm{a}$ & $3.179,1 \mathrm{~b}$ \\
\hline
\end{tabular}

Médias seguidas da mesma letra na coluna não diferem entre si pelo teste de ScottKnott, a $5 \%$ de probabilidade.

Em relação a massa de 100 grãos, observa-se que não houve influência significativa entre os fatores estudados (Tabela 1). Estudos de Giron et al. (2019), utilizando o BRS 423 OL submetido a diferentes arranjos de semeadura $\left(0,90 \mathrm{~m}\right.$ e $20 \mathrm{pl} . \mathrm{m}^{-1} ; 0,73 \mathrm{~m} \times 0,17 \mathrm{~m}$ e 20 pl. $\mathrm{m}^{-1} ; 0,70 \mathrm{~m}$ e 20 pl. $\mathrm{m}^{-1} ; 0,70 \mathrm{~m}$ e $\left.15 \mathrm{pl} . \mathrm{m}^{-1}\right)$, não obtiveram diferença significativa quanto a severidade de mancha preta, massa de 100 grãos e produtividade de vagens, nas condições de Campo Verde-MT.

$\mathrm{Na}$ produtividade de vagens, houve efeito significativa entre os genótipos. Com isso, observa-se que os genótipos BRS 421 OL $(3.941,7$ kg.ha-1), BRS 425 OL (3.886,4 kg.ha-1) e BRS 423 OL (3.690,2 kg.ha-1) obtiveram as maiores produtividades, em comparação ao $1952 \mathrm{OL}(3.179,1$ kg.ha-1) (Tabela 2). Heuert et al. (2019), utilizando o BRS $423 \mathrm{OL}$ submetido a diferentes densidades de semeadura $\left(0,90 \mathrm{~m}\right.$ e 20 pl. $\mathrm{m}^{-1} ; 0,73 \mathrm{~m} \times 0,17 \mathrm{~m}$ e 20 pl.m $\mathrm{m}^{-1} ; 0,70 \mathrm{~m}$ e $\left.15,5 \mathrm{pl} . \mathrm{m}^{-1}\right)$, também não obtiveram diferença significativa quanto a massa de 100 grãos, nas condições de Santo Antônio de Goiás-GO. No entanto, neste mesmo estudo, a produtividade foi superior estatisticamente no arranjo de 0,70 m e 15,5 pl. $\mathrm{m}^{-1}$.

\section{Conclusões}

Nas condições experimentais em que foi desenvolvida esta pesquisa, pode-se concluir que a os arranjos de semeadura não influenciam de forma significativa nas variáveis. No entanto, os genótipos influenciaram na severidade e produtividade, obtendo os melhores resultados com o BRS 
421 OL, BRS 423 OL e BRS 425 OL. Sobretudo, mais estudos são recomendados para confirmação dos resultados obtidos.

\section{Agradecimentos}

Os autores agradecem a Cooperação Técnica estabelecida entre o IFMT Campus São Vicente - Centro de Referência de Campo Verde e a Embrapa, localizada em Santo Antônio do Goiás-GO, vinculado ao projeto SEG 20.18.01.021.00. O agradecimento também é estendido a todas as empresas que aderiram ao Programa de Melhoramento do Amendoim, que estimulam a pesquisa científica e de forma especial a MIAC - Máquinas Agrícolas e Agulhon Agroindustrial de Santo Antônio do Leste-MT. 


\section{Referências}

BERNADES, M. S.; VICCARIO, L. R. Aspectos Fisiológicos do plantio e condução do Seringal em desenvolvimento. In: Simpósio sobre a cultura da Seringueira no Estado de São Paulo, 1986, Piracicaba. Anais... Campinas: Fundação Cargil. p. 193-228.

CONAB. Acompanhamento da Safra Brasileira de Grãos. Safra 2020/21 Sétimo levantamento, v. 8, n. 7, p. 1-117, 2021. Disponível em: <https://www.conab.gov.br/info-agro/safras>. Acesso em: 15 maio 2021.

GAMBA, J. M; PEDELINI, R. Evaluacion del rendimento y calidad de três cultivares de mani. Granoleico, ASEM 484 INTA y ASEM 485 INTA. Xxiv Jornada Nacional de Maní. General Gabrera, Cordoba - Argentina, 2009.

GIRON, F. G.; PEROZINI, A. C.; ARAÚJO, C.; HEUERT, J.; XAVIER, M. F. N.; SUASSUNA, T. M. F.; SANTIN, V. Diferentes espaçamentos e densidades populacionais para cultivar de amendoim BRS 423 em Mato Grosso. In: Anais do Encontro Sobre a Cultura do Amendoim, 16., 2019, Jaboticabal. Anais eletrônicos... Campinas: GALOÁ, 2019.

GIRON, F. G.; HEUERT, J.; PEROZINI, A. C.; XAVIER, M. F. N.; SUASSUNA, T. M. F. Diferentes arranjos de semeadura para a cultivar de amendoim BRS 423 OL nas condições de Campo Verde-MT. South American Sciences, v. 1, n. 2, p. e2010, 2020.

HEUERT, J. et al. Arranjo de semeadura para cultivares decumbentes precoces de amendoim. In: Anais do Encontro Sobre a Cultura do Amendoim, 14., 2017, Jaboticabal. Anais eletrônicos... Campinas: GALOÁ, 2017.

HEUERT, J.; MARTINS, K. B. B.; XAVIER, M. F. N.; BETIOL, R. A. B.; SUASSUNA, T. M. F. Diferentes espaçamentos para a cultivar de amendoim BRS 423 em Goiás. In: Anais do Encontro Sobre a Cultura do Amendoim, 16., 2019, Jaboticabal. Anais eletrônicos... Campinas: GALOÁ, 2019.

NAKAGAWA, J.; NOJIMOTO, T.; ROSOLEM, C. A.; ALMEIDA, A. M.; LASCA, D. H. C. Efeitos da densidade de semeadura da produção de vagens de amendoim. Científica, v. 11, n. 1, p. 79-86, 1983.

NAKAGAWA, J.; LASCA, D. C.; NEVES, J. P. S.; NEVES, G. S.; SANCHEZ, S. V.; BARBOSA, V.; SILVA, M. N.; ROSSETO, C. A. V. Efeito da densidade de semeadura na produção de amendoim. Pesquisa Agropecuária Brasileira, v. 29, n. 10, p. 1547-1555, 1994.

SILVA, F. A. S. E.; AZEVEDO, C. A. V. The Assistat Software Version 7.7 and its use in the analysis of experimental data. African Journal of Agricultural Research, v. 11, n. 39, p. 3733-3740, 2016. 
SUBRAHMANYAM, P.; MCDONALD, D.; GIBBONS, R. W.; NIGAM, S. N.; NEVILL, D. J. Resistance to rust and late leaf spot diseases in some genotypes of Arachis hypogaea. Peanut Science, v. 9, p. 9-14, 1982.

XAVIER, M. F. N.; MEINKE, G. C.; HEUERT, J.; MARTINS, K. B. B.; SUASSUNA, T. M. F. Desempenho agronômico de genótipos de amendoim nas condições de Campo Verde-MT. South American Sciences, v. 1, n. 1, p. e2009, 2020. 